\title{
ANOMALOUS DIFFUSION PROCESSES: STOCHASTIC MODELS AND THEIR PROPERTIES
}

\author{
SEAN CARNAFFAN
}

(Received 16 February 2020; first published online 27 March 2020)

\begin{abstract}
2010 Mathematics subject classification: primary 60G22; secondary 35Q84, 62M09. Keywords and phrases: anomalous diffusion, stochastic processes.
\end{abstract}

Background and motivation. In recent decades, diffusion-like processes whose mean-squared displacement deviates from the linear variance scaling with time exhibited by Brownian motion have been observed routinely in physical experiments. This 'anomalous' behaviour has been observed arising from many different physical mechanisms. For example, particles might be subject to sporadic trapping phenomena, undertake sudden long range relocations, so-called 'Lévy flights', or have correlated increments that either inhibit or exacerbate their spread. Particles exhibiting such behaviour are said to be undergoing anomalous diffusion. In systems of this nature, it is common to observe power variance scaling $\propto t^{\alpha}$ for some exponent $\alpha>0$. The exponent $\alpha$ is used to differentiate classes of anomalous diffusion in that if $\alpha \in(0,1)$, the process is referred to as subdiffusion, if $\alpha \in(1,2)$ superdiffusion, and if $\alpha>2$ it is called hyperdiffusion. Their observation has led to great research interest in their mathematical description, to which this thesis is intended as a contribution. This thesis primarily consists of the contents of four published papers that all contribute significant novel insight into the field of anomalous diffusion processes. Brief summaries of each are as follows.

\footnotetext{
Solving multidimensional fractional Fokker-Planck equations via unbiased density formulas for anomalous diffusion processes. In the first paper, we introduce a novel Monte Carlo method for density estimation of anomalous diffusion processes [2], that by definition doubles as a numerical method for solving generalised Fokker-Planck equations (GFPEs). It invokes the use of tools from Malliavin calculus in its construction, but is elementary to implement. Furthermore, it boasts several

Thesis submitted to the University of Sydney in July 2019; degree approved on 1 October 2019; supervisor Reiichiro Kawai.

(C) 2020 Australian Mathematical Publishing Association Inc.
} 
desirable properties and advantages over existing methods. For example, in contrast to histogram and kernel density based methods, it is unbiased and does not rely on the arbitrary selection of any kernel or bandwidth parameters. Moreover, in the context of GFPEs, solutions are known to often (depending on which integro-differential operator is present) exhibit nondifferentiabilities (or 'cusps') around their mode, a feature that kernel density estimators might struggle to accurately capture in general without an exceedingly large number of Monte Carlo replicates. Our solution method captures the cusping behaviours of the solution accurately, even in higher dimensions and with relatively few Monte Carlo replicates. Furthermore, our estimation procedure is uniformly convergent in the number of Monte Carlo replicates to the true solution on any domain on which the true solution is continuous.

Cusping, transport and variance of solutions to generalised Fokker-Planck equations. In this paper, a mathematical explanation is given for many interesting subtleties in the behaviour of density functions of anomalous diffusion processes. For example, when subdiffusion is induced by sporadic trapping events with lengths drawn from a stable distribution, a cusp was observed at the mode of the corresponding density function. Moreover, when a scalar (positive, without loss of generality) external drift was imposed on the process, the mode of the solution did not 'drift' with time as one might intuitively expect. Instead, the perennially cusped mode remained fixed at the origin for all time, and the right-hand side of the density became more and more spread out. However, when the waiting times were drawn from distributions with less extreme tails (tempered stable and gamma distributions) the cusp, if observed, would come to diminish over time, and the density function seemed to be able to travel in accordance with an externally imposed scalar drift.

These observations were investigated, leading to a set of well-defined mathematical criteria determining when a cusp would appear in the density (and how prominent this cusp would be) and when the density would be able to travel in response to an external drift [1]. These criteria were combined with the variance scaling properties of the relevant mathematical models of subdiffusion to create a method for determining which waiting time distribution was present in experimental data. Moreover, it was shown how the cusping properties of densities could be used to test for the presence of a jump component in experimental data.

Optimal statistical inference for subdiffusion processes. An important aspect of the application of anomalous diffusion processes, as with any stochastic model for real-world phenomena, is calibration of parameters to experimental data. Within the context of subdiffusion, an in-vogue method for parameter estimation is to obtain measures of nonergodicity (disagreement between temporal and spatial averages) of sample trajectories and compare these to theoretical values, reporting the values of the relevant parameters that give the best fit.

This approach has been very successful and has boasted good performance in experiments. However, due to data intensivity constraints of this method, we consider the alternative of classical statistical estimators, since these estimators only require the 
endpoint of each trajectory in their calculation. It is intuitive that performing statistical inference with respect to only a single data point per trajectory will come with a loss of statistical information, since intermediate information about the trajectory is being implicitly disregarded, and therefore, we derive the Fisher information for this sampling regime [4] to quantify the amount of information remaining. The upshot of the analysis is that the information contained in trajectories is nonzero, suggesting that classical estimation procedures might be of benefit in estimating parameters in this context. Moreover, it was found that, when only considering the endpoints of trajectories, the information contained in very short trajectories is equivalent to the information contained in very long ones. This unexpected result suggests that in the best case scenario a great deal of expense could be saved without sacrificing any model calibration performance.

An analytic model for transient anomalous diffusion with highly persistent correlations. Since the introduction of fractional Brownian motion, time series data has been discovered in which the decay of the spectogram implies a Hurst parameter greater that one, suggesting a need to generalise the fractional Brownian motion to capture more persistent correlations. This has been achieved by adapting the moving average integration kernel present in the fractional Brownian motion. The result, termed higher order fractional Brownian motion, was a Gaussian process with the ability to capture highly anomalous spread through its higher order correlation. Concurrently, the prevalence of observations of stochastic processes with jump components led to the application of stable processes to real world data and subsequently the development of tempered stable motion. The key property of the tempered stable motion that makes it useful and unique for time series modelling is the description of crossover dynamics from stable motion on the short-time horizon and Gaussian behaviour on the long-time horizon, which has given it many applications in the literature.

In the final paper, we introduce the higher order fractional generalisation of the tempered stable motion [3]. This created an interesting model in the sense of possessing both highly persistent correlations (allowing it to describe sub-, super- or hyperdiffusion) and transient dynamics from stable to Gaussian marginal distributions. Compared to the higher order stable motion, it also boasts the technical advantage of a finite autocovariance from which many useful properties may be gleaned. We examine some of the technical properties of the process and introduce a simulation scheme for its trajectories. Finally, we perform a Monte Carlo simulation study to illustrate the viability of parameter calibration on experimental data.

\section{References}

[1] S. Carnaffan and R. Kawai, 'Cusping, transport and variance of solutions to generalized FokkerPlanck equations', J. Phys. A 50(24) (2017), Article ID 245001.

[2] S. Carnaffan and R. Kawai, 'Solving multidimensional fractional Fokker-Planck equations via unbiased density formulas for anomalous diffusion processes', SIAM J. Sci. Comput. 39(5) (2017), B886-B915. 
[3] S. Carnaffan and R. Kawai, 'Analytic model for transient anomalous diffusion with highly persistent correlations', Phys. Rev. E 99(6) (2019), Article ID 062120.

[4] S. Carnaffan and R. Kawai, 'Optimal statistical inference for subdiffusion processes', J. Phys. A 52(13) (2019), Article ID 135001.

SEAN CARNAFFAN, School of Mathematics and Statistics, University of Sydney, Camperdown, NSW 2006, Australia e-mail: scarnaffan@gmail.com 